\title{
Lack of association between apolipoprotein C3 gene polymorphisms and risk of coronary heart disease in a Han population in East China
}

\author{
Juan $\mathrm{Yu}^{1,2 \dagger}$, Jingjing Huang ${ }^{1+}$, Yan Liang ${ }^{1 \dagger}$, Baodong Qin ${ }^{1}, \mathrm{Su} \mathrm{He}^{3}$, Jing Xiao ${ }^{3}$, Huimin Wang $^{2}$ and \\ Renqian Zhong ${ }^{1 *}$
}

\begin{abstract}
Background: Several polymorphisms in the apolipoprotein C3 (APOC3) gene have been found association with hypertriglyceridemia(HTG), but the link with coronary heart disease(CHD) risk between ethnicities was still controversial. Among them, reseachers paid more attentions to the promoter polymorphisms T-455C and C-482T because both of them located in insulin-responsive element (IRE) and insulin was thought to exert its action by down-regulating APOC3 gene expression. The aim of this study was to investigate the association of the two polymorphisms of APOC3 with CHD in a Han population in East China.

Methods: TaqMan SNP Genotyping Assays were carried out to detect the genotypes of APOC3 gene, including the T-455C and C-482T, in 286 subjects with CHD and 325 controls without CHD. The levels of serum lipid profiles were also detected by biochemical methods.

Results: There was no difference of genotype frequencies and allele frequencies between the CHD population and the controls $(\mathrm{P}>0.05)$. Compared with the most common genotype $-455 T \mathrm{~T}$ or $-482 \mathrm{CC}$, the variants had neither significantly increased CHD risk, nor the lipid variables showed any statistically relevant differences in the research population. The adjusted OR of CHD were 5.67 [0.27-18.74] and 0.75 [0.20-2.73] in carriers of the APOC3 $-455 \mathrm{C}$ and $-482 T$ variants, respectively $(P>0.05)$. There was also no significant difference in APOC3 haplotype distribution in CHD and controls, but there was a strong linkage disequilibrium between T-455C and C-482T with $\mathrm{D}^{\prime}=0.9293$, 0.8881 , respectively $(P<0.0001)$.
\end{abstract}

Conclusions: Our data did not support a relationship between the two polymorphisms of APOC3 gene and risk of CHD in the Han population in East China.

Keywords: Apolipoprotein C3, Coronary heart disease, Polymorphism, Hypertriglyceridemia

\section{Background}

Both the genetic and environmental factors contribute to the development of coronary heart disease (CHD) which is becoming the leading cause of death in many countries of the world including China. The metabolism of circulating particles such as triglyceride-rich lipoprotein (TRL) was strongly influenced by their content in apolipoprotein C3 (APOC3), a component that inhibits the hydrolysis of

\footnotetext{
* Correspondence: rqzhong@yahoo.com

† Contributed equally

'Department of Laboratory Medicine, Changzheng Hospital, Second Military Medical University and Clinical Immunology Center of PLA, 415 Feng Yang

Road, Shanghai 200003, People's Republic of China

Full list of author information is available at the end of the article
}

these paticles by lipoprotein lipase, and APOE-mediated hepatic uptake of them. By far, data from both clinical evidence $[1,2]$ and mechanism research results $[3,4]$ supported the role of APOC3 in CHD and artherosclerosis (AS), suggesting that the APOC3 was a strong independent risk factor for CHD.

Different genetic and acquired factors impact serum APOC3 concentrations. Five polymorphisms have been found in the APOC3 gene promoter. Among them, two promoter polymorphisms T-455C and C-482T have been studied more extensively because they just located on the insulin-responsive element (IRE) in APOC3 gene. Insulin exerted its action by down-regulating APOC3 gene
C Biomed Central 
expression transcriptionally, however, the presence of mutant sequences seemed to reduce the inhibitory effect of the hormone [5].

Patients with type 2 diabetes mellitus(T2DM) increased levels of circulating APOC3, and it has been linked to enhanced $\beta$-cell apoptosis [6]. Recently we reported that the $\mathrm{T}-455 \mathrm{C}$ or $\mathrm{C}-482 \mathrm{~T}$ variant on the APOC3 gene promoter contributed to an increased risk of HTG in Chinese Han health population, however, no relationship between the two polymorphisms and HTG in T2DM patients was found [7]. Insulin resistance state in T2DM may mask the effect of a common APOC3 haplotype on the risk of HTG. A large body of clinical studies have demonstrated that increased serum triglyceride concentrations were positively associated with risk for CHD $[2,8,9]$, and low APOC3 levels were associated with reduced CHD risk $[10,11]$. Although several studies have supported an association between these two polymorphisms and $\mathrm{CH} \# 1$ [12], others have failed to confirm the relationship [13,14]. Considering the complex of CHD and race difference, what condition is in china? In present study, we investigated the distribution of the two polymorphisms and the relationship to the susceptibility of CHD in the Han Population in East China.

\section{Methods}

\section{Study population}

we selected a total of 611 unrelated adult subjects of both sexes, 286 unrelated Chinese patients (214 males, 72 females, age $56.30 \pm 11.57$ years) with significant coronary stenosis by cardiovascular angiography(according to stenoss $\geq 50 \%$ at least 1 coronary artery)were recruited from Department of cardiolog of Changzheng Hospital, Second Military Medical University of China. 325 controls were selected via health-screening at the Physical Examination Centre or CHD-free of heart disease patients at the same hospital (172 males, 153 females, age $55.79 \pm 12.40$ years). All subjects were of Han ethnicity in East China.

The informed consent was obtained from subjects who agreed to participate in the study, and the study had the approval of the hospital ethics committee.

\section{Biochemical analysis}

The blood sample after 12 hours fasting was collected in tube for measuring. TG, total cholesterol (TC), HDLC and LDLC were measured using the enzymatic method, and APOA1 and APOB were determined by immunonephelometry, and they all measured by automatic biochemistry analyzer (Abbott Aeroset 2000, USA). APOC3 was also measured by automatic biochemistry analyzer (Hitachi 7020, Japan). Samples for APOC3 were measured by a turbidimetric immunoassay, and the reagents were obtained from Sekisui medical co.(Japan) with a precipitant reagent [phosphotungstic acid $0.55 \mathrm{mmol} / \mathrm{l}$ and magnesium chloride $\left(\mathrm{MgCl}_{2}\right) 25 \mathrm{mmol}$. HDL-APOC3 was assayed similarly in the supernatant. TRL-APOC3 was calculated by substracting HDL-APOC3 from total APOC3.

\section{Mutation analysis}

TaqMan SNP Genotyping Assays were carried out to detect the genotypes of APOC3 gene, including the T$455 \mathrm{C}$ and C-482T, in 286 subjects with CHD and 325 controls. There was 20 missing data of genotype due to the template reason(17 in CHD, and 3 in controls). A 2 $\mathrm{ml}$ whole-blood sample containing EDTA was collected for each subject. Genomic DNA was extracted using a Genomic DNA Kit(Axygen Biotechnology, shanghai, China) according to the manufacturer's protocol and store the DNA samples in a $-20^{\circ} \mathrm{C}$ freezer until use. Two valid SNPs rs2854116(T-455C) and rs2854117(C-482T) in the APOC3 gene that are found to be associated with HTG were genotyped. Genotyping was performed applying a TaqMan assay that incorporates minor groovebinding probe technology. A fluorogenic probe consisting of an oligonucleotide labeled with both fluorescent reporter and quencher dyes was included. The primers of rs2854116 are AGAGCTCAGCCCTGTAACCA(forward) and GGGCTTCTTCAGACTTGAGAACAA(reverse), and the probes were VIC-ACTCCAAACATCCCCCMGB and FAM-CTCCAAACACCCCCC-MGB. The primers of rs2854117 were same as rs2854116, but the probes were VIC-CAGAAGACCGGGCATC-MGB and FAM-CAGAAGACCAGGCATC-MGB. All PCR reactions were initially run in a final volume of $5 \mu \mathrm{l}$, comprising of $2.5 \mu \mathrm{l}$ TaqMan genotyping master mix, $0.25 \mu \mathrm{l}$ TaqMan SNP genotyping assay mix $(40 \times), 1 \mu \mathrm{l}$ DNA samples, $1.25 \mu \mathrm{l}$ water. All PCR reactions was carried out in the ABI 7900 with 384 wells, consisting of an initial step of $95^{\circ} \mathrm{C}$ for $5 \mathrm{~min}$, followed by 40 cycles: denaturation at $95^{\circ} \mathrm{C}$ for $15 \mathrm{sec}$, annealing at $60^{\circ} \mathrm{C}$ for $60 \mathrm{sec}$. Amplicon size was $115 \mathrm{bp}$. Genotyping or allele calling was carried out with the ABI Prism 7900HT genetic detection system: SDS 2.3 software. A graph on the computer would show the results of the allelic discrimination plot by automatic allele calling. The horizontal axis indicated an wild allele XX homozygote, the vertical axis indicated mutant allele YY homozygote, and the diagonal was allele $\mathrm{X} / \mathrm{Y}$ heterozygote(Figure 1). When automatic genotyping was not well done, re-testing, or direct sequencing, until got the results. To validate the SNP genotyping assays, $5 \%$ of the genotyped samples were randomly selected for duplication accuracy with a direct sequencing protocol(Sangon Biotech, Shanghai, China) and all of the data for the TaqMan SNP Genotyping Assays and direct sequencing analyses showed perfect concordance. 

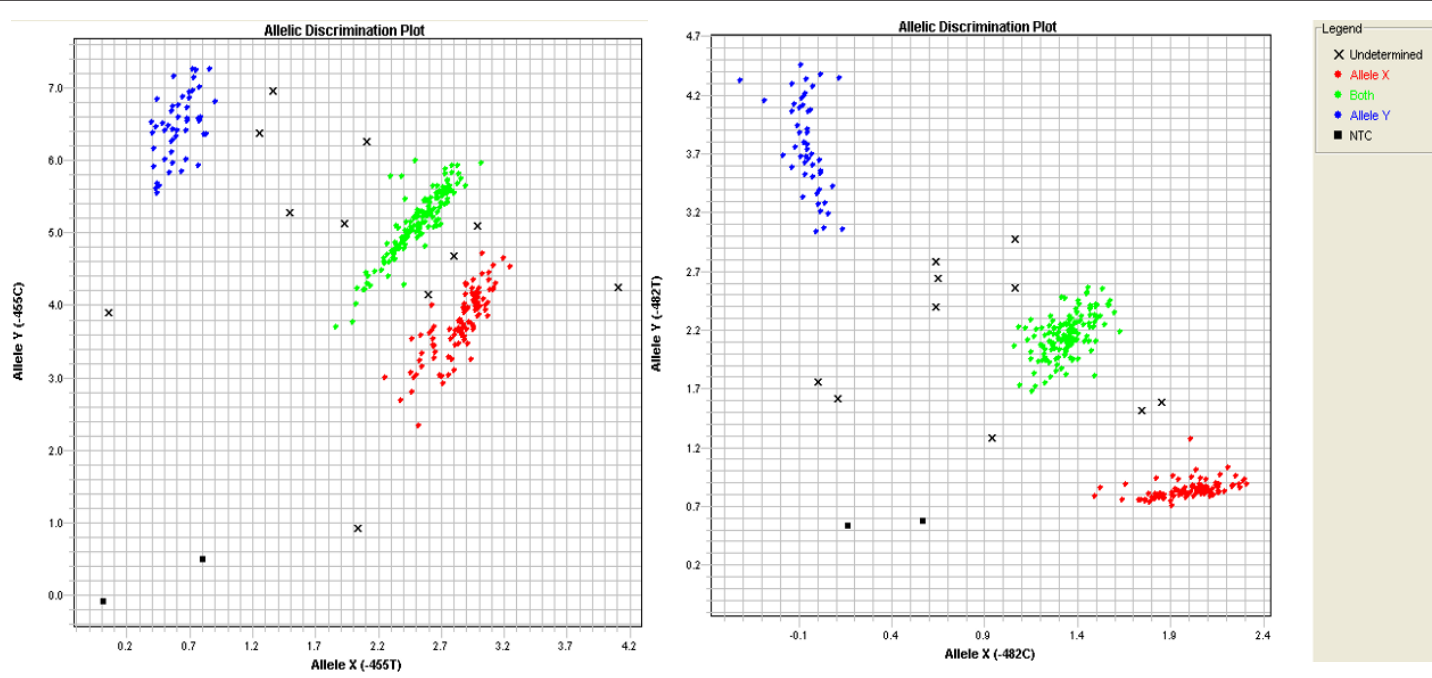

Figure 1 TaqMan SNP Genotyping figure of indicated promoter polymorphisms in APOC3 gene. Left: T-455C, right: C-482T.

\section{Statistical analysis}

Baseline characteristics were shown as mean \pm SD. Due to plasma Lipid concentrations had a skewed distribution, the median and interquartile ranges (Q:difference of Q25 and Q75) were presented: $M \pm Q$. The association between genetic polymorphisms and the prevalence of CHD was assessed using chi-square tests. Odds ratio was determined by comparing each polymorphism (heterozygous, homozygous) to wild type and the $95 \%$ confidence intervals were calculated. Observed frequencies in the CHD and controls were compared to the expected frequencies (Hardy-Weinberg equilibrium) by chi-square test. The distribution of genotype and allele frequencies in CHD and controls were analyzed by chi-square test too. The difference of lipid levels in different genotypes in CHD and controls was evaluated by one-way ANOVA after rank transformation when necessary. Linkage disequilibrium was calculated by Arlequin population genetics analysis software showed in value of D'. All computations were performed by using the stata 8.0 or SAS 9.13 statistical software. Levels of significance were defined as $P<0.05$.

\section{Results}

Genotype and allele frequencies of the APOC3 polymorphic variants for CHD and controls were described in Table 1. The allele frequencies of the rs2854117 $(-482 \mathrm{~T})$ and rs2854116 $(-455 \mathrm{C})$ APOC3 alleles were 0.424 and 0.420 in CHD, 0.407 and 0.410 in controls, respectively. Three polymorphisms were in the HardyWeinberg equilibrium in relevant groups (data not shown). There was no significant difference of the genotype and allele distribution between $\mathrm{CHD}$ and controls $(P>0.05)$.

For the purpose of estimating the impact of the polymorphisms on APOC3 and lipid levels and other accompany complications, genotype-phenotype analysis was performed with data from the CHD and controls. Compared to the wide type $-455 \mathrm{TT}$ or $-482 \mathrm{CC}$, none of the lipid variables and incidence of other accompany complications analyzed showed any statistically relevant differences according to the variant APOC3 promoters in the study population(Table 2 ).

To estimate the CHD risk associated with APOC3 genotypes, logistic regression analysis was performed. Crude and adjusted odds ratios for CHD related to APOC3 -455 and 482 genotypes are shown in Table 3 . The polymorphisms of APOC3 gene promoter were not associated with a significantly increased risk of CHD in the study population.

To estimate the CHD risk associated with APOC3 haplotypes, Arlequin population genetics analysis software

Table 1 Distribution of genotype and allele frequencies of APOC3 in CHD and controls

\begin{tabular}{|c|c|c|c|c|}
\hline & $\mathrm{CHD}(\%)$ & control(\%) & $\chi^{2}$ & $\mathbf{P}$ \\
\hline \multicolumn{5}{|c|}{-455 Genotype frequency } \\
\hline$-455 \pi$ & $90(33.58)$ & 112(34.67) & & \\
\hline$-455 T C$ & $131(48.88)$ & $157(48.61)$ & & \\
\hline$-455 C C$ & $47(17.54)$ & $54(16.72)$ & 0.11 & 0.95 \\
\hline \multicolumn{5}{|c|}{-455 Allele frequency } \\
\hline$-455 \mathrm{~T}$ & $311(58.00)$ & $381(59.00)$ & & \\
\hline$-455 C$ & $225(42.00)$ & $265(41.00)$ & 0.11 & 0.74 \\
\hline \multicolumn{5}{|c|}{-482 Genotype frequency } \\
\hline$-482 C C$ & $89(33.21)$ & $11(34.67)$ & & \\
\hline$-482 \mathrm{CT}$ & $131(48.88)$ & 159(49.23) & & \\
\hline$-482 \pi$ & 48(17.91) & $52(16.10)$ & 0.38 & 0.83 \\
\hline \multicolumn{5}{|c|}{-482 Allele frequency } \\
\hline$-482 C$ & $309(57.60)$ & $383(59.30)$ & & \\
\hline$-482 \top$ & $227(42.40)$ & $263(40.70)$ & 0.32 & 0.57 \\
\hline
\end{tabular}

Numbers in parentheses indicate each frequency of genotype or allele. 
Table 2 Biochemical parameter levels in study population, according to the APOC3 T-455C and C-482T genotypes

\begin{tabular}{|c|c|c|c|c|c|c|}
\hline \multirow[t]{2}{*}{ Parameters } & \multicolumn{3}{|c|}{-455 Genotypes } & \multicolumn{3}{|c|}{-482 Genotypes } \\
\hline & $-455 \mathrm{TT}$ & $-455 \mathrm{TC}$ & $-455 C C$ & -482 CC & $-482 \mathrm{CT}$ & $-482 T T$ \\
\hline Age (years) & $55.64 \pm 16.76$ & $55.54 \pm 16.21$ & $55.48 \pm 14.84$ & $55.22 \pm 16.60$ & $55.56 \pm 16.03$ & $55.39 \pm 15.11$ \\
\hline Male sex (\%) & 65.7 & 62.5 & 60.4 & 66.5 & 61.0 & 63.0 \\
\hline TC $(\mathrm{mmol} / \mathrm{L})$ & $3.92 \pm 1.16$ & $4.00 \pm 1.18$ & $3.84 \pm 1.28$ & $3.91 \pm 1.18$ & $3.99 \pm 1.15$ & $3.87 \pm 1.33$ \\
\hline TG $(m m o l / L)$ & $1.29 \pm 1.05$ & $1.35 \pm 1.17$ & $1.50 \pm 1.20$ & $1.25 \pm 0.98$ & $1.38 \pm 1.25$ & $1.47 \pm 1.12$ \\
\hline $\mathrm{HDLC}(\mathrm{mmol} / \mathrm{L})$ & $1.12 \pm 0.35$ & $1.14 \pm 0.38$ & $1.10 \pm 0.28$ & $1.13 \pm 037$ & $1.13 \pm 0.39$ & $1.09 \pm 0.27$ \\
\hline LDLC (mmol/L) & $2.34 \pm 0.94$ & $2.47 \pm 0.80$ & $2.40 \pm 0.83$ & $2.34 \pm 0.90$ & $2.44 \pm 0.84$ & $2.48 \pm 0.85$ \\
\hline APOAI $(g / l)$ & $1.3 \pm 0.57$ & $1.36 \pm 0.58$ & $1.29 \pm 0.54$ & $1.35 \pm 0.57$ & $1.33 \pm 0.57$ & $1.28 \pm 0.56$ \\
\hline APOB $(g / l)$ & $0.84 \pm 0.29$ & $0.85 \pm 0.31$ & $0.86 \pm 0.31$ & $0.84 \pm 0.27$ & $0.85 \pm 0.31$ & $0.88 \pm 0.35$ \\
\hline APOAI/APOB & $1.69 \pm 0.43$ & $1.83 \pm 0.60$ & $1.68 \pm 0.44$ & $1.69 \pm 0.40$ & $1.84 \pm 0.63$ & $1.68 \pm 0.42$ \\
\hline APOC3(mg/dl) & $8.10 \pm 4.1$ & $8.4 \pm 4.5$ & $7.95 \pm 3.3$ & $8.4 \pm 4.3$ & $8.1 \pm 4.5$ & $8.3 \pm 3.1$ \\
\hline $\begin{array}{l}\text { HDL-APOC3 } \\
(\mathrm{mg} / \mathrm{dl})\end{array}$ & $5.2 \pm 2.4$ & $5.4 \pm 2.2$ & $5.4 \pm 1.8$ & $5.2 \pm 2.2$ & $5.4 \pm 2.2$ & $5.4 \pm 2.0$ \\
\hline $\begin{array}{l}\text { TRL-APOC3 } \\
(\mathrm{mg} / \mathrm{dl})\end{array}$ & $3.35 \pm 3.25$ & $2.8 \pm 2.6$ & $3.5 \pm 3.4$ & $3.2 \pm 3.1$ & $3.0 \pm 2.8$ & $3.35 \pm 3.15$ \\
\hline T2DM (\%) & 20.1 & 20.56 & 26.73 & 18.2 & 21.1 & 28.0 \\
\hline Hypertension (\%) & 44.6 & 44.9 & 51.5 & 41.9 & 45.7 & 54.0 \\
\hline smoker(\%) & 44.9 & 45.5 & 38.4 & 44.7 & 43.5 & 43.2 \\
\hline drinker(\%) & 28.3 & 21.2 & 26.0 & 28.0 & 20.7 & 28.4 \\
\hline
\end{tabular}

Data were shown as mean \pm SD or \%; skewed variables were $\mathrm{M} \pm \mathrm{Q}$. M: median, Q: inter-quartile range, difference of Q25 and Q75.

was applied(Table 4). No relationship was found between haplotypes of APOC3 -455 and -482 and CHD in the study population, but there was a strong linkage disequilibrium between T-455C and C-482T with $\mathrm{D}^{\prime}=0.9293$, 0.8881 , respectively(Table 5).

\section{Discussion}

APOC3, a 79 amino acid glycoprotein synthesized mainly in the liver and to a lesser degree in the intestine, which acted as a constituent of TRL particles and functioned as a key regulator of serum triglyceride levels, inhibiting the lipoprotein lipase-induced hydrolysis of those particles and interfering with liver receptor-mediated lipoprotein uptake. It played a key role in determining the levels in the circulation of potentially atherogenic VLDL and small dense LDL [15]. The results of large clinical studies have indicated that sera APOC3 concentrations were a better predictor of risk for the development and progression of CHD than the traditionally measured TG levels $[1,8]$. APOC3 could also irritate several processes involved in atherogenesis and vascular inflammation. APOC3 stimulated blood-born monocytes and endothelial cells to activate protein kinase $\mathrm{C}$ (PKC) and nuclear factor $\kappa \mathrm{B}(\mathrm{NF}-\kappa \mathrm{B})$ and expression of endothelialproduce vascular cell adhesion molecule-1 (VCAM-1) and intracellular adhesion molecule-1(ICAM-1), and recruitment of monocytes to the vascular wall [16-18]. APOC3 could also activate insulin-resistance pathways in endothelial cells causing endothelial dysfunction [19] and stimulate adipocytes to produce cytokines such as monocyte chemoattractant protein (MCP) 1 and interleukin (IL)6 and suppresses their production of adiponectin [20].

APOC3 was the first lipid-associated gene to be linked by a common polymorphism to hypertriglyceridemia

Table 3 Odds Ratios for CHD according to APOC3 genotypes in the study population

\begin{tabular}{|c|c|c|c|c|}
\hline \multirow[b]{2}{*}{ APOC3 genotypes } & \multicolumn{2}{|c|}{ Unadjusted Model } & \multicolumn{2}{|c|}{ adjusted Model } \\
\hline & $\begin{array}{l}\text { Odds Ratio } \\
\qquad(95 \% \mathrm{Cl})\end{array}$ & $P$ value & $\begin{array}{l}\text { Odds Ratio } \\
(95 \% \mathrm{Cl})\end{array}$ & $P$ value \\
\hline$-455 T$ & 1 & & 1 & \\
\hline$-455 T C$ (-455TT as reference) & $1.00(0.66-1.51)$ & 0.98 & $1.6(0.20-6.81)$ & 0.16 \\
\hline$-455 C C$ (-455TT as reference) & $1.06(0.61-1.88)$ & 0.82 & $5.67(0.27-18.74)$ & 0.26 \\
\hline$-482 C C$ & 1 & & 1 & \\
\hline$-482 \mathrm{CT}$ (-482CC as reference) & $1.00(0.66-1.51)$ & 1.00 & $0.87(0.12-6.25)$ & 0.89 \\
\hline$-482 \pi \mathrm{T}(-482 C \mathrm{C}$ as reference) & $1.06(0.60-1.87)$ & 0.85 & $0.75(0.20-2.73)$ & 0.55 \\
\hline
\end{tabular}

By multiple logistic regression analysis, The multiple logistic-regression model was adjusted for age, gender, presence of hypertension, T2DM, drinking, smoking and all listed lipid leveles. $\mathrm{Cl}$, confidence interval. P values were for the overall comparison among subjects with a given polymorphism and were calculated by chi-square analysis. 
Table 4 APOC3 haplotype distribution in CHD and controls

\begin{tabular}{llllll}
\hline $\mathbf{- 4 5 5}$ & $\mathbf{- 4 8 2}$ & CHD $\mathbf{n}=\mathbf{2 6 9}$ & Controls $\mathbf{n}=\mathbf{3 2 2}$ & $\mathbf{\chi 2}$ & $\mathbf{p}$ \\
\hline 1 & 1 & 0.5109 & 0.5239 & 2.11 & 0.55 \\
1 & 2 & 0.1842 & 0.1486 & & \\
2 & 1 & 0.0821 & 0.0678 & & \\
2 & 2 & 0.2228 & 0.2507 & & \\
\hline
\end{tabular}

1 and 2 stand for common and rare alleles, respectively.

[21]. Genetic variation in the APOC3 gene, particularly in the promoter region, was associated with an increased risk of HTG [5], metabolic syndrome(MS) [22-24] and CHD [12] in some race population. Association between promoter SNPs of APOC3 and plasma triglyceride levels have been consistently reported. The relationship with CHD, however, was more controversial, with some studies indicating a possible link with genetic variability at the locus [12] and others not confirming those associations [13]. In the present study, the association between APOC3 SNPs and CHD was assessed by comparing the SNPs distribution in CHD and controls without CHD. We demonstrated the relationship between the two polymorphisms T-455C and C-482T and CHD in a randomly selected population sample of Han Population in East China. However, neither APOC3 T-455C nor C-482T SNPs were clearly associated with CHD. Together with previous reports, these results indicated that, although APOC3 $\mathrm{T}-455 \mathrm{C}$ or $\mathrm{C}-482 \mathrm{~T}$ variant has a vital impact on plasma triglyceride concentration, this effect did not increase significantly CHD risk.

Further haplotype analyses also did not found any significant frequency difference between CHD and controls, but found there was a strong linkage disequilibrium between $\mathrm{T}-455 \mathrm{C}$ and $\mathrm{C}-482 \mathrm{~T}$.

Ruiz et al reported that cardioprotective effects of promoter common allele $-455 \mathrm{~T}$ or $-482 \mathrm{C}$ of APOC3 gene became dysfunctional in abdominal obesity and hyperglycemia in nonfatal MI [25]. Their research suggested that the effect of the APOC3 $-455 \mathrm{C}$ and $-482 \mathrm{~T}$ haplotype on the risk of nonfatal MI was only evident in lean normoglycemic subjects. In the previous study we also found that the effect of these two variant APOC3 genotype on the risk of HTG was only evident in subjects in healthy population and not in T2DM and speculated that probably because of the existence of a strong insulin resistance in them masked the effects of genetic polymorphism [7]. Indeed, recent studies have shown that patients homozygous for the $-455 \mathrm{C}$

Table 5 linkage disequilibrium analysis of T-455C/C-482T

\begin{tabular}{ccccc}
\hline Group & $\mathbf{n}$ & $\mathbf{D}^{\prime}$ & $\mathbf{r}^{\mathbf{2}}$ & $\mathbf{P}$ \\
\hline CHD & 269 & 0.9293 & 0.1723 & $<0.0001$ \\
Controls & 322 & 0.8881 & 0.2775 & $<0.0001$ \\
Total & 591 & 0.9085 & 0.2251 & $<0.0001$ \\
\hline
\end{tabular}

APOC3 variant were poorly responsive to the APOC3-lowering effects of n-3 PUFAs [26]. CHD patients also suffered from different complications, such as T2DM, Hypertension, abdominal obesity, and Fatty liver et al, they all could mask the effect of a common APOC3 genotype on the risk of CHD. Although we excluded the population of T2DM, there was still no relationship found between them(data not shown). Maybe there was other larval factors we yet not found in CHD mask the association between APOC3 T-455C or C-482T and CHD. Such findings still have other explanations. Firstly, the number of our study samples may be too low to detect a significant association. Secondly, CHD was a multifactorial desease, one or more genetic variations combining with environmental factors may result in phenotypic variability. Thirdly, part of CHD risk attributable to elevated triglyceride levels was due to structural alterations of LDL and HDL, such as oxidation. Fourthly, race differences, genetic background, or environmental differences or methodological flaws or nonrandomized selection of subjects. Finally, a number of conditions such as age, obesity, gender, hormonal status may influence the relationship between APOC3 gene polymorphisms and CHD.

\section{Conclusions}

Altogether with previous and present findings suggested, although APOC3 promoter variants had a key impact on plasma triglyceride and APOC3 levels, this effect didn't raise significantly CHD risk in Chinese Han Population. That was, APOC3 T-455C and C-482T SNPs were not major risk factors of CHD in Han Population in East China. However, other studies were necessary to confirm these results and search for the causing facts.

List of abbreviations

APOC3: apolipoprotein C3; HTG: hypertriglyceridemia; CHD: coronary heart disease; TRL: triglyceride-rich lipoprotein; AS: artherosclerosis; IRE: insulinresponsive element; MCP: monocyte chemoattractant protein; IL: interleukin; T2DM: type 2 diabetes mellitus.

\section{Acknowledgements}

We thank all participants in this study. We also thank Dr. Yang Jing for clinical data collection. We also thank Dr. Wang aihua, Dr. Geng honglian and Dr. Chen jun for their technical supports and constructive suggestions. This work was supported by Hi-Tech research and development program of China (863 Program) [grant number: 2006AA02Z496].

\section{Author details}

'Department of Laboratory Medicine, Changzheng Hospital, Second Military Medical University and Clinical Immunology Center of PLA, 415 Feng Yang Road, Shanghai 200003, People's Republic of China. ${ }^{2}$ Center of Laboratory Medicine, Affiliated Hospital, Nantong University, 20 Xi Si Road, Nantong 226001, People's Republic of China. ${ }^{3}$ Institute of Public Health, Nantong University, 9 Se Yuan Road, Nantong 226001, People's Republic of China.

\section{Authors' contributions}

The study was designed by $R Z, J Y$ and $H W$. JY, JH and $Y L$ equally

contribuited to the work. All subjects data were obtained by $B Q, J H$ and $Y L$.

The database organization was carried out by RZ and HW. Experimental data 
was obtained by $\mathrm{JY}$ and $\mathrm{BQ}$. Data analyses were performed by SH and JX. The paper was written by JY and RZ and all authors read and approved the final manuscript.

\section{Competing interests}

The authors declare that they have no competing interests.

Received: 6 October 2011 Accepted: 4 November 2011

Published: 4 November 2011

\section{References}

1. Olivieri O, Martinelli N, Girelli D, Pizzolo F, Friso S, Beltrame F, Lotto V, Annarumma L, Corrocher R: Apolipoprotein C-III predicts cardiovascular mortality in severe coronary artery disease and is associated with an enhanced plasma thrombin generation. J Thromb Haemost 2010, 8(3):463-471

2. Scheffer PG, Teerlink T, Dekker JM, Bos G, Nijpels G, Diamant M, Kostense PJ, Stehouwer CD, Heine RJ: Increased plasma apolipoprotein CIII concentration independently predicts cardiovascular mortality: the Hoorn Study. Clin Chem 2008, 54(8):1325-1330.

3. Lee HY, Birkenfeld AL, Jornayvaz FR, Jurczak MJ, Kanda S, Popov V Frederick DW, Zhang D, Guigni B, Bharadwaj KG: Apolipoprotein CIII overexpressing mice are predisposed to diet-induced hepatic steatosis and hepatic insulin resistance. Hepatology 2011, 54(5):1650-1660.

4. Kawakami A, Yoshida M: Apolipoprotein CIII links dyslipidemia with atherosclerosis. J Atheroscler Thromb 2009, 16(1):6-11.

5. Li WW, Dammerman MM, Smith JD, Metzger S, Breslow JL, Leff T: Common genetic variation in the promoter of the human apo CIII gene abolishes regulation by insulin and may contribute to hypertriglyceridemia. J Clin Invest 1995, 96(6):2601-2605.

6. Sol EM, Sundsten T, Bergsten P: Role of MAPK in apolipoprotein CIIIinduced apoptosis in INS-1E cells. Lipids Health Dis 2009, 8:3.

7. Yu J, Wang H, Yang S, Yuan J, Chen L, Chen CL, Huang DF, Wang Y, Ju SQ, Zhu J: The Effect of APOC3 Promoter Polymorphisms on the Risk of Hypertriglyceridemia in Chinese Han Population With or Without Type 2 Diabetes Mellitus. Laboratory Medicine 2010, 41(1):34-39.

8. Sacks FM, Alaupovic P, Moye LA, Cole TG, Sussex B, Stampfer MJ, Pfeffer MA, Braunwald E: VLDL, apolipoproteins B, CIII, and E, and risk of recurrent coronary events in the Cholesterol and Recurrent Events (CARE) trial. Circulation 2000, 102(16):1886-1892.

9. Chan DC, Chen MM, Ooi EM, Watts GF: An ABC of apolipoprotein C-III: a clinically useful new cardiovascular risk factor? Int J Clin Pract 2008, 62(5):799-809.

10. Pollin Tl, Damcott CM, Shen H, Ott SH, Shelton J, Horenstein RB, Post W, McLenithan JC, Bielak LF, Peyser PA: A null mutation in human APOC3 confers a favorable plasma lipid profile and apparent cardioprotection. Science 2008, 322(5908):1702-1705.

11. Hofker MH: APOC3 null mutation affects lipoprotein profile APOC3 deficiency: from mice to man. Eur J Hum Genet 2010, 18(1):1-2

12. Olivieri O, Stranieri C, Bassi A, Zaia B, Girelli D, Pizzolo F, Trabetti E, Cheng S, Grow MA, Pignatti PF: ApoC-III gene polymorphisms and risk of coronary artery disease. J Lipid Res 2002, 43(9):1450-1457.

13. Dallongeville J, Cottel D, Montaye M, Codron V, Amouyel P, Helbecque N: Impact of APOA5/A4/C3 genetic polymorphisms on lipid variables and cardiovascular disease risk in French men. Int J Cardiol 2006 , 106(2):152-156.

14. Sentinelli F, Romeo S, Maglio C, Incani M, Burza MA, Scano F, Coccia F, Cossu E, Leonetti F, Baroni MG: Lack of effect of apolipoprotein C3 polymorphisms on indices of liver steatosis, lipid profile and insulin resistance in obese Southern Europeans. Lipids Health Dis 2011, 10(1):93.

15. Sacks FM, Zheng C, Cohn JS: Complexities of plasma apolipoprotein C-III metabolism. J Lipid Res 2011, 52(6):1067-1070.

16. Kawakami A, Aikawa M, Alcaide P, Luscinskas FW, Libby P, Sacks FM: Apolipoprotein CIII induces expression of vascular cell adhesion molecule- 1 in vascular endothelial cells and increases adhesion of monocytic cells. Circulation 2006, 114(7):681-687.

17. Kawakami A, Aikawa M, Libby P, Alcaide P, Luscinskas FW, Sacks FM: Apolipoprotein CIII in apolipoprotein B lipoproteins enhances the adhesion of human monocytic cells to endothelial cells. Circulation 2006 , 113(5):691-700.
18. Kawakami A, Aikawa M, Nitta N, Yoshida M, Libby P, Sacks FM: Apolipoprotein CIII-induced THP-1 cell adhesion to endothelial cells involves pertussis toxin-sensitive $\mathrm{G}$ protein- and protein kinase $\mathrm{C}$ alphamediated nuclear factor-kappaB activation. Arterioscler Thromb Vasc Biol 2007, 27(1):219-225.

19. Kawakami A, Osaka M, Tani M, Azuma H, Sacks FM, Shimokado K, Yoshida M: Apolipoprotein CIII links hyperlipidemia with vascular endothelial cell dysfunction. Circulation 2008, 118(7):731-742.

20. Abe Y, Kawakami A, Osaka M, Uematsu S, Akira S, Shimokado K, Sacks FM, Yoshida M: Apolipoprotein CIII induces monocyte chemoattractant protein-1 and interleukin 6 expression via Toll-like receptor 2 pathway in mouse adipocytes. Arterioscler Thromb Vasc Biol 2010, 30(11):2242-2248.

21. Rees A, Stocks J, Sharpe CR, Vella MA, Shoulders CC, Katz J, Jowett NI, Baralle FE, Galton DJ: Deoxyribonucleic acid polymorphism in the apolipoprotein A-1-C-III gene cluster. Association with hypertriglyceridemia. J Clin Invest 1985, 76(3):1090-1095.

22. Onat A, Hergenc G, Sansoy V, Fobker M, Ceyhan K, Toprak S, Assmann G: Apolipoprotein C-III, a strong discriminant of coronary risk in men and a determinant of the metabolic syndrome in both genders. Atherosclerosis 2003, 168(1):81-89.

23. Pollex RL, Ban MR, Young TK, Bjerregaard P, Anand SS, Yusuf S, Zinman B, Harris SB, Hanley AJ, Connelly PW: Association between the $-455 T>C$ promoter polymorphism of the APOC3 gene and the metabolic syndrome in a multi-ethnic sample. BMC Med Genet 2007, 8:80

24. Zheng C, Khoo C, Furtado J, Sacks FM: Apolipoprotein C-III and the metabolic basis for hypertriglyceridemia and the dense low-density lipoprotein phenotype. Circulation 2010, 121(15):1722-1734.

25. Ruiz-Narvaez EA, Sacks FM, Campos H: Abdominal obesity and hyperglycemia mask the effect of a common APOC3 haplotype on the risk of myocardial infarction. Am J Clin Nutr 2008, 87(6):1932-1938.

26. Olivieri O, Martinelli N, Sandri M, Bassi A, Guarini P, Trabetti E, Pizzolo F, Girelli D, Friso S, Pignatti PF, Corrocher R: Apolipoprotein C-III, n-3 polyunsaturated fatty acids, and "insulin-resistant" T-455C APOC3 gene polymorphism in heart disease patients: example of gene-diet interaction. Clin Chem 2005, 51(2):360-367.

doi:10.1186/1476-511X-10-200

Cite this article as: Yu et al.: Lack of association between apolipoprotein C3 gene polymorphisms and risk of coronary heart disease in a Han population in East China. Lipids in Health and Disease 2011 10:200.

\section{Submit your next manuscript to BioMed Central and take full advantage of:}

- Convenient online submission

- Thorough peer review

- No space constraints or color figure charges

- Immediate publication on acceptance

- Inclusion in PubMed, CAS, Scopus and Google Scholar

- Research which is freely available for redistribution

Submit your manuscript at www biomedcentral.com/submit
C Biomed Central 